Microwave Engineering with Wireless Applications 


\section{Other titles of interest to engineers}

B. Allen, Analogue Electronics for Higher Studies

G.J. Awcock and R. Thomas, Applied Image Processing

Rodney F.W. Coates, Underwater Acoustic Systems

C.W. Davidson, Transmission Lines for Communications, second edition

Peter J. Fish, Electronic Noise and Low Noise Design

M.E. Goodge, Analog Electronics

Robin Holland, Microcomputer Fault-finding and Design, second edition

Paul A. Lynn, An Introduction to the Analysis and Processing of Signals, third edition

Paul A. Lynn, Radar Systems

R.C.V. Macario, Cellular Radio - Principles and Design, second edition

R.J. Mitchell, Microprocessor Systems - An Introduction

Noel M. Morris, Electrical Circuit Analysis and Design

M.S. Nixon, Introductory Digital Design

F.J. Owens, Signal Processing of Speech

Dennis N. Pim, Television and Teletext

M. Richharia, Satellite Communications Systems - Design Principles, second edition

P. Rohner, Automation with Programmable Logic Controllers

P.R. Shepherd, Integrated Circuit Design Fabrication and Test

M.J.N. Sibley, Optical Communications, second edition

P. Silvester, Electric Circuits

Andrew Simmonds, Data Communications and Transmission Principles

P.M. Taylor, Robotic Control

Trevor J. Terrell and Lik-Kwan Shark, Digital Signal Processing

M.J. Usher and C.G. Guy, Information and Communication for Engineers

M.J. Usher and D.A. Keating, Sensors and Transducers, second edition

G.S. Virk, Digital Computer Control Systems

L.A.A. Warnes, Analogue and Digital Electronics

L.A.A. Warnes, Electrical and Electronic Engineering, second edition

B.W. Williams, Power Electronics - Devices, Drivers, Applications and Passive Components, second edition 


\title{
Microwave Engineering with Wireless Applications
}

\author{
S.R. Pennock and P.R. Shepherd
} School of Electronic and Electrical Engineering University of Bath 
C) S.R. Pennock and P.R. Shepherd 1998

Softcover reprint of the hardcover 1st edition 1998 978-0-333-72801-7

All rights reserved. No reproduction, copy or transmission of this publication may be made without written permission.

No paragraph of this publication may be reproduced, copied or transmitted save with written permission or in accordance with the provisions of the Copyright, Designs and Patents Act 1988, or under the terms of any licence permitting limited copying issued by the Copyright Licensing Agency, 90 Tottenham Court Road, London W1P 9HE.

Any person who does any unauthorised act in relation to this publication may be liable to criminal prosecution and civil claims for damages.

The authors have asserted their rights to be identified as the authors of this work in accordance with the Copyright, Designs and Patents Act 1988.

First published 1998 by MACMILLAN PRESS LTD

Houndmills, Basingstoke, Hampshire RG21 6XS

and London

Companies and representatives

throughout the world

ISBN 978-1-349-14763-2 ISBN 978-1-349-14761-8 (eBook)

DOI 10.1007/978-1-349-14761-8

A catalogue record for this book is available from the British Library

This book is printed on paper suitable for recycling and made from fully managed and sustained forest sources.

$\begin{array}{llllllllll}10 & 9 & 8 & 7 & 6 & 5 & 4 & 3 & 2 & 1\end{array}$

$\begin{array}{llllllllll}07 & 06 & 05 & 04 & 03 & 02 & 01 & 00 & 99 & 98\end{array}$ 


\section{Contents}

1 Transmission Lines 1

1.1 Introduction .................... 1

1.2 Transmission Line Basics . . . . . . . . . . . . . . . . . . 1

1.2.1 Equivalent Circuit ................ 1

1.2.2 Derivation of the Wave Equation and its Solution ..... 2

1.2.3 The Propagation Constant ............ 4

1.2.4 Phase and Group Velocities ............ 5

1.3 Characteristic Impedance. . . . . . . . . . . . . 7

1.4 Reflections .................... 8

1.4.1 Variation in Reflection with Distance Along Line . . . . 9

1.4.2 Variation in $Z_{\text {in }}$, Total Voltage and Total Current with Distance ................... 10

1.5 The Smith Chart . . . . . . . . . . . . . . . . 12

1.6 Voltage Standing Wave Ratio (VSWR) . . . . . . . . . . 13

1.6.1 Driving Point Impedance Along the Line . . . . . . . . 19

1.7 Lossy Lines . . . . . . . . . . . . . . . . . . . . . . . . . . . . . . . . . . .

1.8 Examples ............................. 20

1.9 Microwave Transmission Line Technologies . . . . . . . . . . . 22

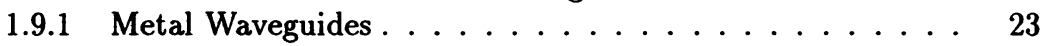

1.9 .2 Coaxial Line . . . . . . . . . . . . . . . . . 24

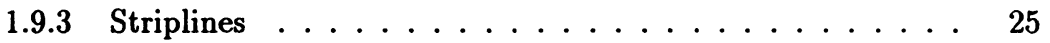

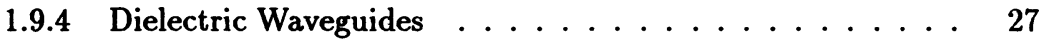

1.10 Summary ....................... 28

2 Electromagnetic Waves 29

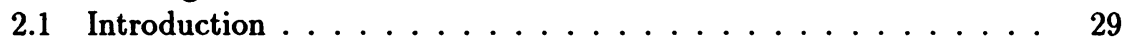

2.2 Field Definitions . . . . . . . . . . . . . . . . 29

2.3 Poynting Vector . . . . . . . . . . . . . . . 30

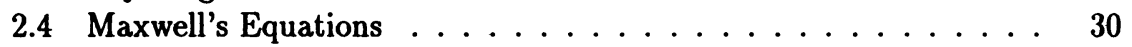

2.5 Solution to Maxwell's equations . . . . . . . . . . . . 31

2.6 Plane Waves . . . . . . . . . . . . . . . . . . . . . 33

2.6.1 Plane Wave Fields and Characteristic Impedance . . . . 35

2.7 Boundary Conditions . . . . . . . . . . . . . . . 38

2.8 Normal Incidence at a Dielectric/Dielectric Boundary . . . . . . . 39

2.8.1 Comparisons with Transmission Lines . . . . . . . . . 41 
2.9 Conductor/Dielectric Boundary . . . . . . . . . . . . . 41

2.10 Oblique Incidence at Dielectric/Dielectric Boundary . . . . . . . . 42

2.10.1 Oblique Propagation . . . . . . . . . . . . . . . 42

2.10 .2 Oblique Incidence . . . . . . . . . . . . . . . . 43

2.11 Propagation Examples . . . . . . . . . . . . . . . . 45

2.11.1 Radomes and Lenses . . . . . . . . . . . . . . . 47

2.11.2 Radiowave Propagation and Fading . . . . . . . . . . 49

2.12 Summary .................... 50

3 Matching $\mathbf{5 3}$

3.1 Introduction . . . . . . . . . . . . . . . 53

3.2 Quarter Wave Transformer . . . . . . . . . . . . . . 53

3.3 Reactive Matching . . . . . . . . . . . . . 55

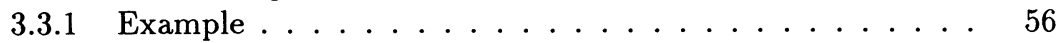

3.4 Impedance Setting . . . . . . . . . . . . . . . 58

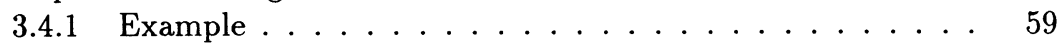

3.5 Single Stub Matching . . . . . . . . . . . . . . . . . . 60

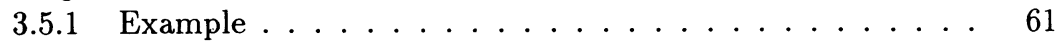

3.6 Double Stub Matching . . . . . . . . . . . . . . . . 62

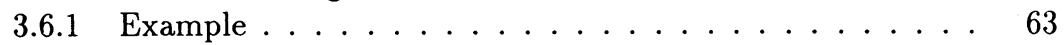

3.7 Broadband Matching . . . . . . . . . . . . . . . 65

3.7.1 Multiple Section Transformers ........... 65

3.7.2 Maximally Flat Transformers . . . . . . . . . . . 66

3.7.3 Equal Ripple Transformers . . . . . . . . . . . . 67

3.8 Summary . . . . . . . . . . . . . . . . 69

4 Matrix Representation of Circuits and Signal Flow Graphs $\quad 71$

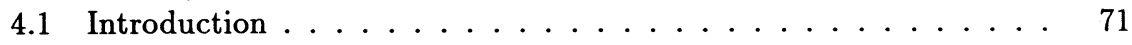

4.2 ABCD or Chain Matrix . . . . . . . . . . . . 71

4.2.1 Examples of ABCD Matrices ............ 73

4.2.2 Cascading Two-Ports . . . . . . . . . . . . . 75

4.2.3 Reflection and Insertion Loss ........... . . . 76

$4.3 \mathrm{Z}$ and $\mathrm{Y}$ Matrices . . . . . . . . . . . . . . . . . . . . 78

4.4 Scattering Matrix . . . . . . . . . . . . . . . . 79

4.4.1 Definitions of Variables and Parameters . . . . . . . . 79

4.4.2 Two-Port $S$-Parameters with Standard Reference Impedances 82

4.4.3 Relation Between ABCD and $S$-Parameters . . . . . 84

4.5 Scattering Transfer Parameters ..................... 84

4.6 Physical Restrictions . . . . . . . . . . . . . . . . . 85

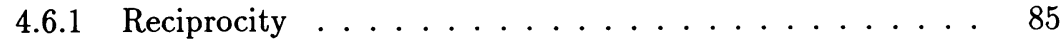

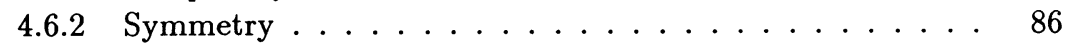

4.6 .3 Losslessness ... . . . . . . . . . . . . . 86

4.7 Construction of Signal Flow Graphs . . . . . . . . . . . . . 87

4.7.1 Example of Graph Construction . . . . . . . . . . 88

4.7.2 Rules for Simplifying Flow Graphs . . . . . . . . . . 91 
4.7.3 Mason's Non-Touching Loop Rule . . . . . . . . . . . . 92

4.7.4 Mason's Rule Applied to S-Parameter Networks . . . . . . 93

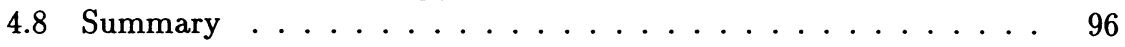

5 Waveguides 99

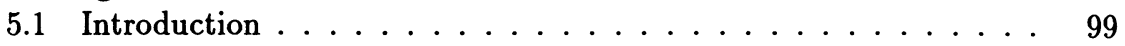

5.2 Parallel Plate Waveguide . . . . . . . . . . . . . 100

5.2 .1 TM Case . . . . . . . . . . . . . . . . . . . 101

$5.2 .2 \quad$ TE Case . . . . . . . . . . . . . . . . . 102

5.2.3 Phase Constant and Mode Spectrum . . . . . . . . . 102

5.3 Mode Orthogonality . . . . . . . . . . . . . . . . . . . 104

5.4 Arbitrary Fields in Waveguides . . . . . . . . . . . . . 107

5.5 Rectangular Waveguide . . . . . . . . . . . . . . 108

5.5.1 Characteristic Impedance . . . . . . . . . . . . . . 109

5.5.2 Cross Sectional Field Dependence . . . . . . . . . . . 110

5.5.3 Propagation Constant and Mode Spectrum . . . . . . . . 111

5.5.4 Power Flow in Rectangular Waveguide . . . . . . . . . . . 114

5.5.5 Power Loss in Waveguide . . . . . . . . . . . . . . . . 114

5.6 Waveguide Iris . . . . . . . . . . . . . . . . . 117

5.6.1 Other Waveguide Discontinuities . . . . . . . . . 120

5.7 Circular Guides . . . . . . . . . . . . . . . . . . . 121

5.7.1 Modes in Coaxial Cable . . . . . . . . . . . . . . 123

5.8 Dielectric Slab Waveguide . . . . . . . . . . . . . . . . . . 124

5.8 .1 Bound Modes . . . . . . . . . . . . . . . . . . 125

5.8.2 Mode Spectra of Dielectric Slabs . . . . . . . . . . 128

5.8.3 Analysis by the Transverse Resonance Technique . . . . . 128

5.8 .4 TM Modes . . . . . . . . . . . . . . . . . . . . . 130

5.9 Two Dimensional Cross Sections . . . . . . . . . . . . . . . . 131

5.9 .1 Slab Loaded Waveguide . . . . . . . . . . . . . 132

5.9 .2 Finline . . . . . . . . . . . . . . . . . 134

5.9.3 Rectangular Dielectric Image and Insular Guides . . . . 134

5.9.4 Inset Dielectric Guide (IDG) . . . . . . . . . . . . 136

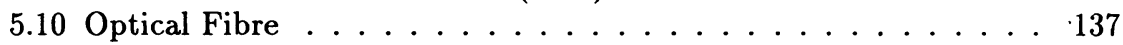

5.11 Summary . . . . . . . . . . . . . . . . 139

6 Microstrip 141

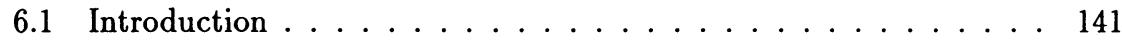

6.2 Microstrip Geometries . . . . . . . . . . . . . . . . . 141

6.3 Moding . . . . . . . . . . . . . . . . . . . 143

6.4 Basic Parameters . . . . . . . . . . . . . . . . 145

6.4.1 Example Calculations . . . . . . . . . . . . . . . 148

6.5 Dispersion in Microstrip . . . . . . . . . . . . . . . . . . 148

6.5.1 Variation of $Z_{o}$ with Frequency . . . . . . . . . . 149

6.5.2 Other Frequency Limitations . . . . . . . . . . . . . 150

6.6 Losses in Microstrip . . . . . . . . . . . . . . . . . . 151 
6.6.1 Conductor Loss . . . . . . . . . . . . . . 151

6.6 .2 Dielectric Loss . . . . . . . . . . . . . . . . . 151

6.6.3 Parasitic Losses . . . . . . . . . . . . . . . . . 152

6.6.4 Example Calculations . . . . . . . . . . . . 152

6.7 Summary ...................... 153

7 Passive Devices $\quad \mathbf{1 5 5}$

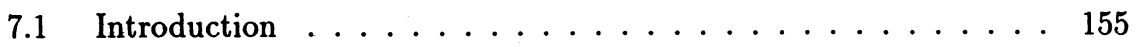

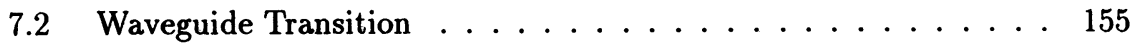

7.3 Waveguide Attenuators and Phase Shifters . . . . . . . . . 156

7.4 Slots in Waveguide ... . . . . . . . . . . . . . . 157

7.4 .1 Slotted Line . . . . . . . . . . . . . . . . 158

7.4 .2 Slot Antennas . . . . . . . . . . . . . . 159

7.5 Waveguide Sliding Short Circuit . . . . . . . . . . . 160

7.6 Two-hole Directional Coupler . . . . . . . . . . . . 161

7.7 Coupled Line Analysis . . . . . . . . . . . . . . . . . . . . . . . . . . . . . . 163

7.7.1 Alternative Coupling Condition .......... 168

7.8 Circuit Junctions . . . . . . . . . . . . . . . . 169

7.8 .1 T-junctions . . . . . . . . . . . . 169

7.8.2 Magic T and Hybrid Ring . . . . . . . . . . . 170

7.8.3 Branch Line Coupler .............. . . 171

7.8.4 Wilkinson Coupler ................ 172

7.9 Microwave Cavities and Resonators . . . . . . . . . . . 172

7.9.1 Waveguide Cavity ... . . . . . . . . . . 173

7.9.2 Cavity Equivalent Circuit and Varactor Tuning . . . . 175

7.9.3 Dielectric Resonator Cavity . . . . . . . . . . . 178

7.10 Lumped Impedance Elements . . . . . . . . . . . . . 179

7.10.1 Short Line Inductor/Capacitor . . . . . . . . . . . . 180

7.10 .2 Spiral Inductor .................. 181

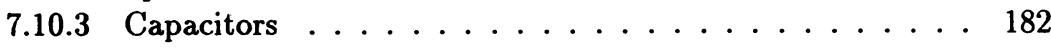

7.11 Microwave Filters . . . . . . . . . . . . . . 183

7.11.1 Butterworth and Chebyshev Synthesis Equations . . . . 183

7.11.2 Basic Transmission Line Filters . . . . . . . . . . 185

7.11.3 Short Line Filter Implementation . . . . . . . . . 186

7.11 .4 Bias Networks . . . . . . . . . . . . . . . 189

7.12 Summary ..................... 190

8 Active Devices 193

8.1 Introduction . . . . . . . . . . . . . . . 193

8.2 P-N Junction . . . . . . . . . . . . . . . . . 193

8.2.1 Reverse Bias .................. . . . 194

8.2 .2 Forward Bias ..................... 194

8.2.3 Equivalent Circuit ................. 196

8.2.4 The Diode at Microwave Frequencies . . . . . . . . . 197

8.3 PIN Diode . . . . . . . . . . . . . . . . 197 
8.3.1 Current Flow and Series Resistance of the PIN Diode . . . 198

8.3.2 Switching the PIN Diode . . . . . . . . . . . . 199

8.4 Tunnel and Back Diodes . . . . . . . . . . . . . . . . 200

8.5 Schottky Barrier Diode . . . . . . . . . . . . . . . . . 202

8.6 IMPATT Diode . . . . . . . . . . . . . . . . . . 204

8.7 Gunn Diode . . . . . . . . . . . . . . . . . 206

8.8 Microwave Transistors . . . . . . . . . . . . . . . 207

8.8.1 Bipolar Junction Transistor (BJT) . . . . . . . . . . 208

8.8.2 Metal Semiconductor Field Effect Transistor . . . . . . . . 209

8.8.3 High Electron Mobility Transistors (HEMT) . . . . . . . . 210

8.8.4 Packaged Transistors . . . . . . . . . . . . . . . 210

8.9 Non-linear Effects . . . . . . . . . . . . . . . . 211

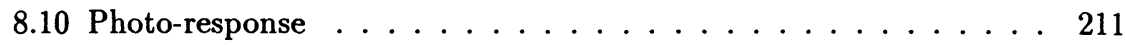

8.11 Summary .......................... 212

9 Detectors and Mixers 213

9.1 Introduction . . . . . . . . . . . . . . . . 213

9.2 Detector Diode . . . . . . . . . . . . . . . 213

9.2.1 Detector Diode Current Sensitivity . . . . . . . . . . . 214

9.3 Noise Equivalent Power and the Tangential Signal Sensitivity . . . 218

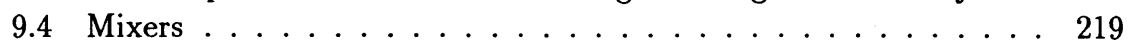

9.4.1 Mixer Components . . . . . . . . . . . . 221

9.4.2 Mixer Parameters ................. 222

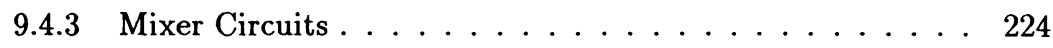

9.5 Summary ............................. 228

10 Control Circuits $\quad 229$

10.1 Introduction . . . . . . . . . . . . . . . . . . . . . . . . . . . . . . 229

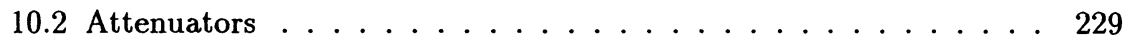

10.2.1 Single Diode Circuits . . . . . . . . . . . . . . . 229

10.2.2 $\mathrm{Pi}$ and $\mathrm{T}$ Attenuators . . . . . . . . . . . . . 231

10.3 Limiters . . . . . . . . . . . . . . . . . . . . . . . . . . . . . . . . . . . . . . 233

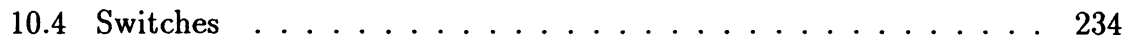

10.4.1 Single Diode Switch .............. . . . . 235

10.4.2 Isolation and Bandwidth Extension . . . . . . . . . 238

10.5 Phase Shifters . . . . . . . . . . . . . . . . 239

10.5.1 Reflection Based Phase Shifters . . . . . . . . . . 240

10.5.2 Switched Path . . . . . . . . . . . . . . . 244

10.5.3 Switched Filter or Hi/Lo Phase Shifter . . . . . . . . 245

10.6 Summary . . . . . . . . . . . . . . . 249

11 Amplifiers and Oscillators 251

11.1 Introduction . . . . . . . . . . . . . . . . . 251

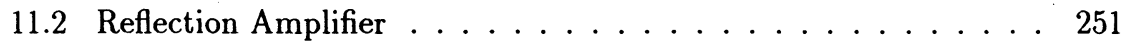

11.2.1 Oscillation and Gain Conditions .......... 252 
11.3 Parametric Amplifier . . . . . . . . . . . . . . 255

11.3.1 Manley-Rowe Power Relations . . . . . . . . . . 255

11.4 Two Port and Transistor Amplifier . . . . . . . . . . . 259

11.4 .1 Network Stability . . . . . . . . . . . . . . 259

11.4.2 Amplifier Gain . . . . . . . . . . . . . . . 261

11.4.3 Amplifier Noise . . . . . . . . . . . . . . . . . . 263

11.4.4 Effects of Package Parasitics and Matching . . . . . . 265

11.5 Active Isolator . . . . . . . . . . . . . . . . . . . 265

11.6 Transistor Oscillator . . . . . . . . . . . . . . . . . 267

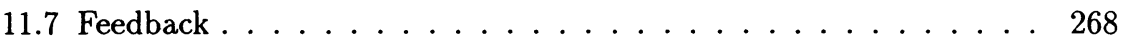

11.7 .1 Series Feedback . . . . . . . . . . . . . . . 268

11.7.2 Shunt Feedback . . . . . . . . . . . . . . . . 269

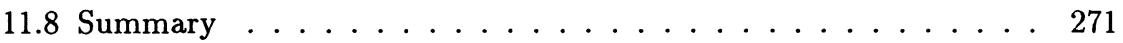

12 Microwave Measurements 273

12.1 Introduction . . . . . . . . . . . . . . . 273

12.2 Frequency Measurement . . . . . . . . . . . . . . . 274

12.2.1 Wavemeters . . . . . . . . . . . . . . . 274

12.2.2 Frequency Counters . . . . . . . . . . . . . 276

12.2.3 Spectrum Analysers . . . . . . . . . . . . . . . 278

12.3 Power Measurement . . . . . . . . . . . . . . . . 279

12.3.1 Power Head Elements _. . . . . . . . . . . . . . 280

12.3.2 Power Head Match . . . . . . . . . . . . . . . 280

12.3.3 Uncertainty Due to Multiple Reflections . . . . . . . . 282

12.3.4 Power Head Calibration Factor and Efficiency . . . . . . 282

12.4 Noise and Measurement . . . . . . . . . . . . . . 283

12.4.1 Sources of Noise . . . . . . . . . . . . . . 283

12.4.2 Equivalent Noise Parameters . . . . . . . . . . . . 286

12.4 .3 Noise Figure . . . . . . . . . . . . . . . . . . 288

12.4.4 Cascaded Stages _. . . . . . . . . . . . . 290

12.4.5 Noise Figure Measurement . . . . . . . . . . . . . . . 291

12.4 .6 Noise Sources . . . . . . . . . . . . . . . . . . . . 292

12.4.7 Automatic Noise Figure Measurement . . . . . . . . 293

12.5 Reflection and Transmission Measurement . . . . . . . . 295

12.5.1 Slotted Line . . . . . . . . . . . . . . . . . . 295

12.5.2 Reflectometers . . . . . . . . . . . . . . 296

12.5.3 Transmission Measurement . . . . . . . . . . . . . 297

12.6 Network Analysers . . . . . . . . . . . . . . . 300

12.6.1 Calibration Techniques . . . . . . . . . . . . 301

12.6.2 Through-Reflect-Line (TRL) Calibration . . . . . . . . 307

12.7 Time Domain Reflectometry . . . . . . . . . . . . . 308

12.8 Summary . . . . . . . . . . . . . . . . 308 
13 Antennas $\quad 311$

13.1 Introduction . . . . . . . . . . . . . . 311

13.2 Antenna Characterisation ............... 311

13.2.1 Polar Patterns . . . . . . . . . . . . . . . 311

13.2.2 Antenna Characterisation Parameters . . . . . . . . . . 312

13.3 Antenna System Power Budget . . . . . . . . . . . . . . . . 314

13.3.1 Friis Transmission Formula . . . . . . . . . . . . 314

13.3.2 Radar Equation . . . . . . . . . . . . . . . . . . 315

13.4 Short Current Filament Antenna . . . . . . . . . . . . . 317

13.4.1 Magnetic Vector Potential . . . . . . . . . . . . 317

13.4.2 Fields of the Short Current Filament . . . . . . . . . . 318

13.4.3 Far-field Region of the Short Current Filament . . . . . . . 320

13.4.4 Characterisation of the Short Filament . . . . . . . . . . 320

13.4.5 Distributed Source Antennas . . . . . . . . . . . . 322

13.5 Array Antennas . . . . . . . . . . . . . . . . . . 326

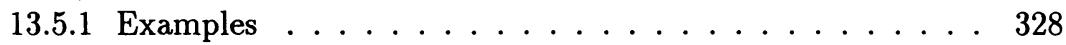

13.5.2 Major Beams ................. . . . 329

13.6 Antenna Images . . . . . . . . . . . . . . . . . . . . . . . . . . . . . . 331

13.7 Summary ...................... 332

A Appendix $\mathbf{3 3 5}$

A.1 Vector Calculus . . . . . . . . . . . . . . . . . 335

A.1.1 Vector Identities . . . . . . . . . . . . . 336

A.1.2 Integration identities . . . . . . . . . . . . 336

A.2 Boundary Conditions . . . . . . . . . . . . . . . 336

A.2.1 Simplifications. . . . . . . . . . . . . 338

A.3 Poynting Vector . . . . . . . . . . . . . . . . . . . 338

A.4 Circuit Matrix Relationships . . . . . . . . . . . . . . 339

A.4.1 $[\mathrm{S}]$ and $[\mathrm{Z}] \ldots \ldots \ldots \ldots \ldots . \ldots . \ldots . \ldots 339$

A.4.2 $[\mathrm{S}]$ and $[\mathrm{Y}] \ldots \ldots \ldots \ldots \ldots \ldots . \ldots \ldots$

A.4.3 [S] and T-matrix or ABCD Matrix ... . . . . . . 340

A.4.4 [S] and Wave Transmission Parameters [T] . . . . . . . 340

A.5 Separation of Variables . . . . . . . . . . . . . . . . 341

A.5.1 Cartesian Co-ordinates . . . . . . . . . . . . 341

A.5.2 Cylindrical Co-ordinates .............. . . 341

A.5.3 Appendix: Möbius or Bilinear Transformation . . . . . . . 342

$\begin{array}{ll}\text { References } & \mathbf{3 4 5}\end{array}$

$\begin{array}{ll}\text { Index } & \mathbf{3 4 8}\end{array}$ 


\section{Preface}

Necessity is said to be the mother of invention and it was necessity that played a large part in the creation of this book. The authors have been teaching high frequency engineering to undergraduate students for some years. Finding suitable texts to support these lecture courses has been a problem. Microwave engineering texts tend to fall into two categories. Firstly there are the specialist texts, dealing in great detail with one particular aspect, and costed well beyond the typical resources of most University students. Secondly there are the more affordable texts which are broadly based, but not so comprehensive. Their treatment of many topics is insufficient to give the student a good grounding in the subject, and do not lead to a point where circuits and systems can be realised. As a result we felt that we could not actually recommend any books for the students to purchase, instead - we provided a reading list of books which were obtainable from the library, and which together covered the syllabus material to a reasonable extent. Often the students relied entirely on the lecture notes we provided.

Clearly there was a requirement for a reasonably comprehensive introductory book to high frequency and microwave engineering, which went into sufficient depth in the various topics, provided good background information to the student but still remained affordable. This is a very tough target to reach, but we believe that the text you are holding largely meets these requirements.

Microwaves are playing a larger and more important role in everyday life. We define the region of microwaves to be between $1 \mathrm{GHz}$ and $100 \mathrm{GHz}(30 \mathrm{~cm}$ to $3 \mathrm{~mm}$ free space wavelength). The main applications are in the fields of communications and radar systems. The boom in mobile radio communications has meant that extra bandwidth is required and the high frequency spectrum is becoming overcrowded. Hence mobile telephones now operate in the microwave region. Indeed, many of the new future developments in the communications field will occur at microwave frequencies as the majority of lower frequencies are already in use. In conjunction with wireless local area networks operating at microwave and millimetre wave frequencies for connection of computing and other equipment, the term wireless has been revived and has again taken on great importance in modern engineering. This fact is reflected in the subtitling of this book.

The figure below shows a generalised microwave heterodyne transmitter/receiver system, showing the typical components which make up the system. In outlining the structure of this book, we shall refer to this system, illustrating the relevance of each chapter on these components and sub-systems.

Chapter 1 is concerned with transmission lines. The transmission line is the connecting line between the various components in the system, and comes in many different forms depending on particular technology and application. This chapter introduces the particular problems of high frequency analysis where the signal wavelengths are of the same order of magnitude as, or smaller than, the circuit dimensions. These problems necessitate a different approach from normal circuit analysis, involving the concepts of characteristic impedance and reflection of power. 


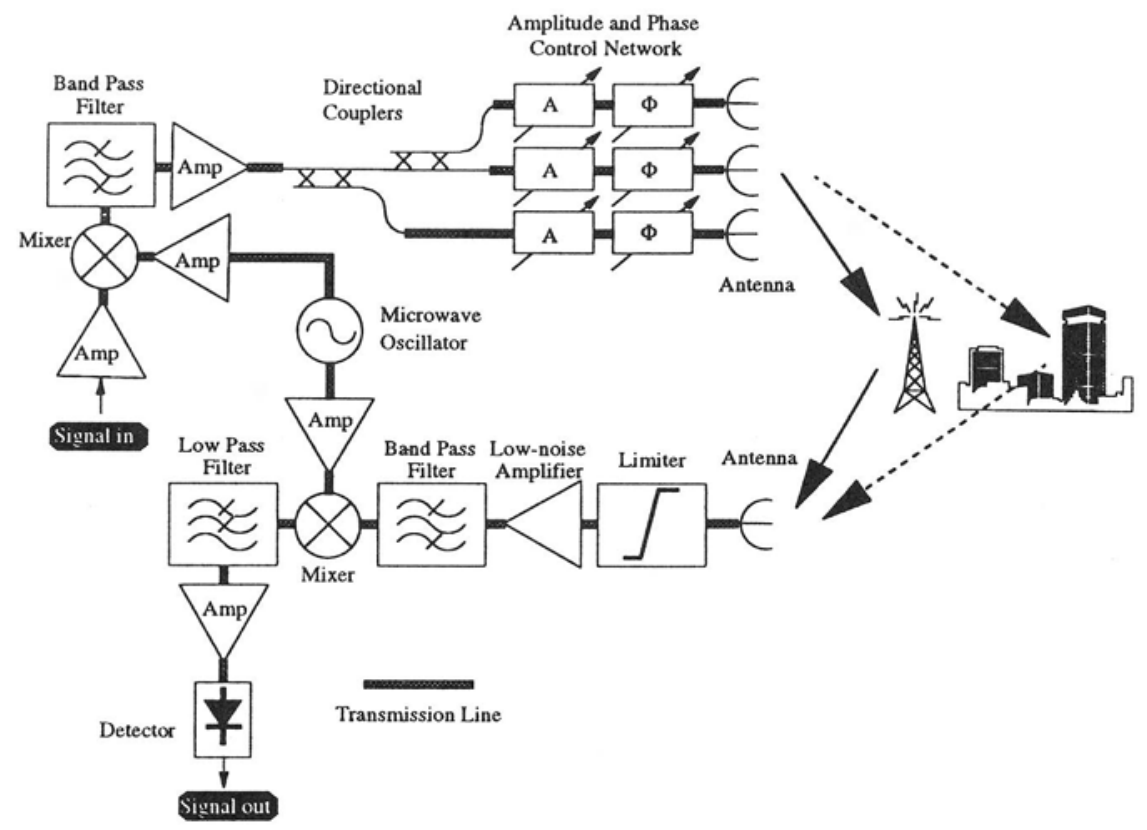

A typical microwave heterodyne transmitter/receiver system

In many situations we need to consider the transmission of microwave signals in terms of electromagnetic wave concepts, rather than voltages and currents. Chapter 2 is concerned with the description of electromagnetic waves, and their analysis provides the basis for the investigation of many effects. These include signal propagation in free space, the reflection of signals from targets (as illustrated in the diagram) and to the behaviour of transmission lines such as waveguides.

Chapter 3 is concerned with matching techniques. The reflection effects described in Chapter 1 can lead to non-optimum circuit performance and even damage to components. It is vital that components are well matched and this chapter describes the design techniques for achieving these matching conditions. This effects all the components and interconnections in the illustrated system.

Chapter 4 is concerned with two commonly used analysis tools, the matrix representation of microwave circuits, and signal flow diagrams. Once the circuit has been analysed, it is often a simple task to invert the analysis to provide a design process for the circuit. These techniques can be applied to any of the linear components in the system, such as amplifiers, filters and phase shifters.

The next two chapters concentrate on two specific types of transmission line which are the most commonly used. Firstly Chapter 5 deals with waveguide structures, which are more commonly used for high power and low loss transmission. Consequently they are typically used in the connections to the transmitting 
antenna in the system example, and may even form the antenna itself.

A very commonly used transmission line structure is microstrip and this is dealt with in Chapter 6. This is a planar structure, very similar in form to printed circuit boards, but requiring a greater dimensional tolerance and higher specification dielectric support. These are light-weight, small structures but which are limited to relatively low power levels. In the illustrated system, microstrip is likely to be used as the transmission line connections between and within such circuits as the receiver amplifiers, filters, mixers, etc.

Chapter 7 concerns itself with passive devices such as attenuators, filters and phase shifters. As can be seen from the figure, such devices occur in different parts of the system, requiring different specifications. Hence this chapter deals with the basic configurations available and the design approaches which can be used to meet a given specification.

Chapter 8 is concerned with the various control devices such as diodes and transistors and their modelling to derive equivalent circuits. These components are then used in the active circuits such as mixers, detectors and amplifiers, the design of which are described in the next few chapters.

Chapter 9 deals with detectors and mixers, which are based on diodes. Again these occur in different parts of the system and again have different specifications and configurations.

Chapter 10 is concerned with control circuits such as limiters, attenuators, switches and phase shifters based on the active elements described in Chapter 8.

Chapter 11 deals with amplifiers and oscillators, which are mainly based on transistors. The chapter describes the different forms available and the design approaches required.

Chapter 12 is concerned with measurements at microwave frequencies. The measurement parameters include frequency, power, noise and reflection and transmission parameters. The particular difficulties of, and special equipment required for, microwave measurements are detailed. In terms of the example system, all the individual components and interconnections will have to be characterised at some stage in the system development.

Chapter 13 deals with antennas, as illustrated in the example system these act as both transmitters and receivers of microwave signals. The chapter describes the main antenna structures in common use, defines the characteristic terms which specify the performance of antennas, and provides the transmission and radar equations required for overall system design.

We would like to thank the authors of the University of Leeds Summer School on Microwave Subsystem Design for permission to use some of the material from their course note book. Finally we would like to dedicate this book to our wives, Debs and Barbara, for putting up with us while we prepared it.

\section{S. R. PENNOCK \& P. R. SHEPHERD}

